\title{
Restricciones financieras del desarrollo económico: teoría y políticas para los países en desarrollo
}

\author{
Jennifer Hermann
}

RESUMEN

En este artículo se analizan las restricciones financieras del desenvolvimiento económico en los países en desarrollo desde los puntos de vista teórico y normativo. A partir de una interpretación keynesiana, se deduce la necesidad de políticas de financiamiento que alivien esas restricciones, ante la natural propensión del sistema financiero a actuar de manera poco funcional para el desarrollo económico. Luego se proponen tres líneas de política, teniendo en cuenta las características específicas de los países en desarrollo: políticas de asignación de recursos, focalizadas en los segmentos estratégicos para el desarrollo económico y financiero; políticas de control de la fragilidad financiera y externa, y políticas compensatorias, de carácter más intervencionista, en particular los programas de crédito dirigido, privado o público, como complemento de las políticas de asignación de recursos y las barreras reglamentarias de tipo anticíclico para reforzar el control de dicha fragilidad. 


\section{I}

\section{Introducción}

La importancia del dinamismo financiero para el desarrollo económico está ampliamente reconocida y se ha comprobado de forma empírica en innumerables trabajos sobre el tema. La historia demuestra, sin embargo, que no hay un modelo único o ideal de sistema financiero para fomentar el desarrollo económico y que apenas ha habido países que fueran capaces de combinar el desarrollo financiero y el económico. Solo pueden citarse como ejemplos los casos de Alemania, los Estados Unidos de América y el Reino Unido de Gran Bretaña e Irlanda del Norte, los dos últimos, con un sistema financiero basado en el mercado de capitales, y el primero con uno sobre la base del crédito privado (Zysman, 1983). Los demás países cuya industrialización se aceleró en la posguerra, entre ellos los países en desarrollo estudiados en este artículo, fundamentaron ese proceso en alguna combinación de crédito público y exterior, y lograron diversos grados de éxito que mostraron el desajuste entre la dinámica de sus sistemas financieros y las necesidades de financiamiento del desarrollo económico.

A pesar de que resulta difícil generalizar en este tema, es posible determinar rasgos comunes de países en niveles semejantes de desarrollo. Entre los países industrializados, las mayores dificultades se concentran en el financiamiento de la innovación - tanto en la fase de investigación y desarrollo (I+D), como en la de implementación de nuevos procesos (Hall, 2002; Matouk, 2010) — debido al mayor riesgo de esas inversiones. En los países en desarrollo, los desafíos impuestos por la propia condición de rezago y por la competencia de economías más avanzadas crean dificultades adicionales. Por una parte, la necesidad de recursos es especialmente aguda en varios sectores. Por otra, el atraso se extiende también a los sistemas financieros que, generalmente, son limitados y poco diversificados (Gershenkron, 1962; Furtado, 1976). En esos casos, el desarrollo económico puede verse directamente restringido por la inadecuación del sistema financiero nacional.

Este artículo se enmarca en ese debate y en él se intenta contribuir, en el plano teórico y en el normativo, a la comprensión y determinación de las restricciones financieras del desenvolvimiento económico en los países en desarrollo. En el plano teórico, se propone una interpretación keynesiana, inspirada en dos de sus vertientes: la neokeynesiana, aquí representada por las contribuciones de J. Stiglitz (Stiglitz y Weiss, 1981; Stiglitz, 1994), y la postkeynesiana, apoyada en las obras de J. M. Keynes (1943, 1937a y 1937b) y H. Minsky (1982 y 1986) sobre el tema. A partir de esa interpretación, en el plano normativo se propone un elenco básico de política financiera, con el objetivo de ampliar el grado de funcionalidad del sistema financiero para el desarrollo económico, teniendo en cuenta las características específicas de los países que procuran alcanzarlo.

En la sección II, la discusión se inicia con una síntesis del debate teórico respecto del papel del sistema financiero en el desenvolvimiento económico. En las secciones siguientes se aplica el enfoque keynesiano al análisis de los países en desarrollo, centrándose en tres aspectos: sus necesidades específicas de financiamiento del desarrollo económico (véase la sección III), las condiciones de funcionalidad del sistema financiero para este proceso (véase la sección IV) y el perfil de política financiera que se precisa para mejorar esa funcionalidad (véase la sección V). En la sección VI se presentan las conclusiones del artículo.

\section{II}

\section{Sistema financiero y desarrollo económico}

J. Schumpeter (1934) y J.M. Keynes (1943, 1937a y 1937b) colocaron los cimientos del debate teórico con respecto al papel macroeconómico del sistema financiero. El primero resaltó la relevancia del crédito para el desarrollo económico y Keynes señaló que el mercado monetario era el principal condicionante de la dinámica de la economía, tanto a corto como a largo plazo.

A partir de los años cincuenta, este debate ganó impulso con la publicación del conocido artículo de Gurley y Shaw (1955), seguido por otras contribuciones 
significativas: Gurley y Shaw (1960); Shaw (1973); McKinnon (1973); Stiglitz (1994) y Minsky (1982 y 1986), entre otros. El primer artículo citado dio origen al modelo Gurley-Shaw y los dos siguientes al modelo Shaw-McKinnon, ambos de filiación teórica neoclásica y brevemente resumidos en la siguiente subsección. Los dos últimos, descritos posteriormente con más detalle (ya que constituyen el fundamento de las políticas propuestas en este estudio), constituyen las principales referencias teóricas del enfoque keynesiano, en sus vertientes neokeynesiana y postkeynesiana, respectivamente.

\section{Breve síntesis de la visión neoclásica}

En el modelo Gurley-Shaw, se sostiene que un sistema financiero diversificado que ofrezca formas variadas de asignación del ahorro favorece el crecimiento económico. Se propone una especie de ley de Say para el mercado financiero: la oferta de activos rentables, con características diversas, tiende a atraer parte del ahorro agregado, creando así su propia demanda. De esa forma, la demanda de moneda y las tasas de interés de equilibrio se reducen y se incrementa la oferta de fondos para financiar inversiones. Ese enfoque, sin embargo, no analiza las condiciones y políticas que favorecen el desarrollo financiero. Esa cuestión solo se trata en el modelo Shaw-McKinnon.

El modelo Shaw-McKinnon se basa en tres hipótesis fundamentales (Fry, 1995; Hermann, 2003): i) el financiamiento del crecimiento económico requiere ahorro; ii) el ahorro agregado es una función positiva de la tasa de interés real, y iii) los mercados financieros libres hacen que la tasa de interés real, el ahorro agregado y, por extensión, el crecimiento económico lleguen a sus niveles "óptimos".

Las dos primeras hipótesis son meras aplicaciones de la conocida teoría monetaria neoclásica. La tercera sintetiza la hipótesis de mercados eficientes (Malkiel, 1994), según la cual —en ausencia de barreras exógenas para la libre elección de los actores- las tasas de interés reflejan correctamente los fundamentos micro y macroeconómicos de los correspondientes activos.

Según este enfoque, cualquier segmento del mercado se vuelve viable si tiene libertad para establecer los precios de los activos según sus fundamentos. Una situación de restricción al financiamiento del desarrollo económico no se interpreta como síntoma de mal funcionamiento del sistema financiero, sino de la economía en que se inscribe ese sistema.

Así pues, para la formación de un mercado financiero completo se recomiendan: i) políticas de liberalización destinadas a evitar que la normativa establezca obstáculos que impidan el ajuste de los precios a las condiciones de retorno y riesgo de los activos; ii) supervisión del sector (para la seguridad del ahorrador individual), y iii) políticas macroeconómicas centradas en la estabilidad monetaria, de modo que ese riesgo se mantenga en niveles reducidos. De acuerdo con este enfoque, tales políticas estimulan la demanda de activos (a través de la reducción de eventos), la oferta (mediante la diminución de costos) y la diversificación del mercado (gracias a la eliminación de barreras reglamentarias), con lo que se promueve el desarrollo financiero.

\section{El enfoque keynesiano}

El enfoque keynesiano del sistema financiero tiene como base una postura crítica con respecto a la hipótesis de los mercados eficientes. En la modalidad neokeynesiana, esa hipótesis se sustituye por la de los fallos del mercado, es decir, las condiciones estructurales que, incluso en situaciones de libre mercado, impiden el ajuste de los precios a los fundamentos económicos. En el mercado financiero suele señalarse que el fallo principal es la asimetría de información entre los potenciales deudores y acreedores ${ }^{1}$. Debido a la asimetría de información, los bancos no pueden distinguir adecuadamente los diversos niveles de riesgo de los posibles deudores para establecer tasas de interés eficientes en relación con cada proyecto. Ante esa limitación, establecen tasas homogéneas para proyectos diferentes en cuanto al riesgo. De esta situación se deriva una primera fuente de ineficiencia: las tasas de interés de equilibrio del mercado de los activos no reflejan correctamente sus fundamentos microeconómicos, porque el acreedor de la operación no conoce perfectamente esos fundamentos. Al no resultar posible determinar cada riesgo, los bancos aplican dos criterios tradicionales de evaluación: i) a mayores tasas de retorno les corresponden niveles más altos de riesgo (Tobin, 1958), y ii) la tasa de interés aceptada por el deudor es, a la vez, un indicador de sus expectativas de retorno y de su propensión al riesgo. Por lo tanto, aunque los acreedores prevean riesgos elevados, incorporarlos integralmente a las tasas de interés no sería una solución, debido al efecto de selección adversa (atracción de deudores más propensos al riesgo e inhibición de los más

\footnotetext{
${ }^{1}$ Suelen señalarse otros fallos de mercado, como por ejemplo, Stiglitz, 1994; BID, 2005, cap. 11: los costos de transacción, los costos de información, las externalidades positivas y negativas, los mercados incompletos y la competencia imperfecta. A excepción del último, los demás son inherentes al mercado financiero.
} 
conservadores). De este modo, los acreedores potenciales tienden a protegerse racionando el crédito y creando una cierta falta de completitud del mercado en segmentos que suponen una mayor dificultad de evaluación o de compensación de los riesgos ${ }^{2}$. Los casos más frecuentes de racionamiento y falta de completitud se producen:

i) en el mercado de capitales, ante el alto riesgo para inversionistas no profesionales;

ii) en los financiamientos a largo plazo y, en general, de las innovaciones, cuyos riesgos son de difícil previsión;

iii) en el crédito a las pequeñas y medianas empresas (pymes), $y$

iv) en el crédito a la población con bajo nivel de ingresos, considerada de alto riesgo, como consecuencia de sus niveles más reducidos de rentas y garantías, dando lugar, en muchos países, a una situación de exclusión financiera. La indiscutible relevancia de estos segmentos para el desarrollo económico pone de manifiesto otra fuente de ineficiencia del sistema financiero: la tendencia a actuar de forma poco funcional para este proceso.

El enfoque postkeynesiano es aún más radical en la crítica a la hipótesis de los mercados eficientes. Desde ese punto de vista, el principal obstáculo para la eficiencia de los mercados es la incertidumbre que existe en torno de todas las decisiones económicas y, especialmente, las transacciones financieras (Carvalho, 2010; Hermann, 2011a). Como observó Kregel (1980), el problema no se limita al acceso a la información y a su costo, sino a la propia existencia, en el momento decisivo, de los indicadores futuros necesarios para las estimaciones de retorno y riesgo de los activos. En consecuencia, al contrario de lo que sucede con el riesgo, la incertidumbre no se puede cuantificar, es decir, no se puede contabilizar en las tasas de interés del mercado. Esto justifica la existencia habitual de un cierto grado de preferencia por la liquidez, como medio de protección contra riesgos imprevisibles.

En opinión de Keynes, la preferencia por la liquidez es la principal fuente de ineficiencia macroeconómica en la asignación de recursos, tanto en el mercado de activos, donde crea una tendencia cortoplacista que dificulta (y encarece) el financiamiento de las inversiones, como en el mercado de bienes, en el que constituye la principal causa de las recesiones. Este

2 Se considera que un mercado es incompleto cuando uno o varios segmentos teóricamente posibles no existen en la práctica, por desinterés por parte de la oferta o de la demanda. hecho refuerza las dificultades anteriormente señaladas para el financiamiento del desarrollo económico. Según el enfoque postkeynesiano, esas dificultades reflejan, a mayor escala, las dificultades de financiamiento de la inversión agregada. Keynes indicó que este financiamiento se produce en dos etapas. La primera, que denominó de finance o financiamiento inicial, consiste en la demanda de crédito de las empresas para iniciar nuevas inversiones y puede satisfacerse con créditos a corto plazo. Sin embargo, para que la inversión se complete, las empresas deben ser capaces de emitir títulos a plazos más largos (incluidas las acciones), compatibles con el plazo del activo que se quiere financiar. Keynes denominó funding o financiamiento a largo plazo a esta etapa.

Como observó Kregel (1986), la etapa de financiamiento inicial no es obligatoria, ya que la inversión se puede financiar directamente mediante la emisión de acciones o de deuda a largo plazo. Este caso, sin embargo, es menos probable que el sugerido por Keynes. En las dos etapas, lo que hace que la inversión sea viable es un entorno de baja (o decreciente) preferencia por la liquidez (que reduce las tasas de interés de equilibrio del mercado de activos), pero el grado requerido de renuncia a la liquidez es, obviamente, mayor en la fase de financiamiento a largo plazo. Esto tiene consecuencias en el costo del financiamiento: los intereses exigidos para los títulos a largo plazo por los ahorradores e instituciones financieras que podrían adquirirlos son, como norma general, mayores que los de los títulos a corto plazo. Es decir, la curva de rendimientos (estructura a plazo de las tasas de interés) normalmente es ascendente (Cargill, 1983; Hermann, 2011b). Este hecho hace que la segunda fase sea más dificultosa que la de financiamiento inicial y supone una mayor penalización para las actividades que requieren financiamiento a largo plazo.

En la fase de financiamiento inicial, los actores decisivos son los bancos comerciales que, al captar depósitos a la vista (pasivo a cortísimo plazo), también concentran sus activos en operaciones a corto plazo. Una vez atendida esa demanda, la inversión produce nuevos ingresos y, a partir de estos, más ahorro. Aunque el valor del ahorro sea idéntico al de las nuevas inversiones (Keynes, 1937a), ello no garantiza el financiamiento a largo plazo, ya que en un entorno de alta preferencia por la liquidez, el ahorro se asignará predominantemente a activos a corto plazo (incluido el efectivo). En esa fase es necesario que las instituciones financieras no bancarias tengan interés y sean capaces de atraer nuevos ahorros para activos a mediano y largo plazo.

En resumen, según el enfoque keynesiano, los actores decisivos en el proceso de financiamiento de las 
inversiones no son los ahorrantes (las empresas y familias que mantienen recursos en el sistema financiero), sino las instituciones financieras: los bancos comerciales en la fase de financiamiento inicial y las demás instituciones financieras (incluidos los bancos universales) en la fase de financiamiento a largo plazo (Carvalho, 1997). Esa interpretación justifica el papel fundamental que el enfoque postkeynesiano atribuye al sistema financiero como condicionante del desarrollo económico.

Más allá de la preferencia por la liquidez, la incertidumbre justifica lo que Keynes (1943, cap. 12) llamó el comportamiento convencional: seguir a la mayoría en los momentos de mayor dificultad de evaluación de riesgos. Como consecuencia de este comportamiento, el mercado tiende a exacerbar los movimientos de apreciación o depreciación de los activos, produciendo burbujas especulativas en el primer caso y crisis de deflación de activos en el segundo. Minsky (1982 y 1986) añade a ese análisis la hipótesis de fragilidad financiera, resaltando que: i) las fases de crecimiento económico siempre están acompañadas de un aumento del endeudamiento; ii) el endeudamiento tiende a ser mayor cuanto más desarrollado esté el sistema financiero; iii) las expectativas que motivan la asunción de esas deudas están rodeadas de incertidumbre; iv) la frustración de esas expectativas puede poner en peligro los pagos previstos, lo que genera un riesgo de crisis de liquidez en el sistema financiero, y v) la confirmación de esas expectativas, sin embargo, no constituye necesariamente una garantía de seguridad para el mercado, porque tiende a prolongar, a veces indebidamente, las fases optimistas, de tal forma que se generan burbujas especulativas cuyo estallido desencadena crisis financieras (Kregel, 1997).

La fragilidad financiera de las economías de mercado tiene, por lo tanto, causas estructurales: la incertidumbre inherente a las relaciones del sistema financiero y su desarrollo. El grado de fragilidad de la economía en cada período dependerá de las condiciones de los contratos de deuda que se firmen. En relación con este aspecto, Minsky propone una taxonomía de los deudores, clasificándolos como: i) operadores de cobertura, cuyos ingresos esperados superan los gastos con las deudas contraídas; ii) especuladores, cuyos ingresos esperados —en la fase inicial del contrato— solo cubren los gastos de intereses, siendo necesario refinanciar el principal, y iii) los sistemas Ponzi, utilizados por unos especuladores incluso más propensos al riesgo, que esperan refinanciar el principal y los intereses en la fase inicial. Los especuladores (incluidos los que emplean un sistema Ponzi) actúan con un mayor grado de fragilidad porque, además del riesgo de frustración de las expectativas de ingresos, dependen de nuevos créditos para cubrir el desajuste de plazos entre su activo (con plazo más largo) y su pasivo (con plazo más corto).

Así, el grado de fragilidad financiera de la economía se ve incrementado por dos factores: el nivel de endeudamiento de los actores y la proporción de deudores especuladores. El primero refleja el ritmo de crecimiento económico y el grado de desarrollo financiero del país, cuyos avances tienden a ampliar el acceso de los actores a fuentes externas de financiamiento. En cuanto al segundo factor, una proporción elevada de deudas especulativas no constituye una anomalía, sino un rasgo común que refleja la dificultad crónica para obtener financiamiento a largo plazo en las economías de mercado, lo que conduce a las empresas al endeudamiento a corto plazo para financiar activos de vencimiento a largo plazo. De este modo, la fragilidad del sistema financiero es inherente a su desarrollo.

En resumen, en la perspectiva postkeynesiana, el sistema financiero tiende a actuar de forma doblemente disfuncional con respecto al desarrollo económico: la falta de completitud del mercado en los segmentos con mayor incertidumbre se suma a la tendencia a la fragilidad financiera.

En los enfoques de filiación keynesiana se justifica la actuación del Estado en el sistema financiero de diversas formas. Además de la supervisión del sector, sugerida en el enfoque neoclásico, se recomiendan políticas de regulación prudencial (restricciones de la libre asignación de recursos), desde incentivos hasta asignaciones específicas (a los sectores prioritarios) y, ante las dificultades de financiamiento del desarrollo, también formas más intervencionistas de actuación por medio de políticas de crédito (privado) dirigido y de crédito público (en este último caso, esas políticas pueden requerir incluso la creación de bancos públicos de desarrollo). 


\section{III}

\section{Financiamiento del desarrollo económico}

\section{Características del proceso de desarrollo}

El desenvolvimiento económico es un fenómeno complejo, que requiere un crecimiento prolongado del producto interno bruto (PIB) unido a transformaciones estructurales en la economía. Ese proceso tiene lugar mediante una serie de inversiones en nueva capacidad productiva, en dos sentidos: se amplía el acervo de capital y se comienza a utilizar nuevos tipos de capital físico y humano. De esta forma, el desarrollo incluye siempre algún grado de innovación, que se lleva a cabo mediante la introducción de nuevos productos, sectores de actividad, procesos productivos y patrones de consumo (Schumpeter, 1934).

Un movimiento generalizado de expansión de las inversiones, como el que caracteriza cada fase del desarrollo económico, requiere algún tipo de aporte complementario al autofinanciamiento. Esta condición básica es el fundamento de la relevancia del desarrollo financiero para el desenvolvimiento económico, siendo reconocida por las diversas corrientes teóricas que tratan el tema. La complejidad del proceso de desarrollo, sin embargo, tiene consecuencias significativas para la comprensión y determinación de sus necesidades de financiamiento (Studart, 2005; Carvalho, 2010; Hermann, 2011a). Entre las mayores dificultades, cabe resaltar:

i) El elevado volumen de capital requerido: esto dificulta el acceso de muchas empresas a recursos de terceros, que resultan escasos o costosos.

ii) La presencia de innovaciones: los nuevos sectores, productos y procesos productivos no cuentan con un historial de lucro acumulado, lo que — además de exponer a las empresas innovadoras al racionamiento del crédito - limita también su capacidad de autofinanciamiento.

iii) Externalidades: una parte de las inversiones necesarias para el progreso del proceso de desarrollo se destina a la expansión de la infraestructura productiva y urbana, en muchos casos con perfil de bien público (con un retorno social mayor que el retorno microeconómico esperado), lo que limita el interés privado.

iv) El papel del consumo: el mantenimiento del proceso de expansión de las inversiones y de las innovaciones requiere que también se expanda el consumo doméstico. Tal como ocurre con las inversiones, el financiamiento de ese consumo a mayor escala difícilmente podrá apoyarse solo en recursos propios, aunque esos recursos tiendan a aumentar como consecuencia de la expansión del empleo y, posiblemente, de algunos progresos en el perfil de la distribución de los ingresos. De esta forma, unas condiciones favorables de acceso al crédito por parte de las familias constituyen un complemento importante para la estructura de financiamiento del desarrollo económico.

v) Posibles efectos sobre la balanza comercial: algunos de los efectos de encadenamiento (hacia delante y hacia atrás) producidos por las nuevas inversiones pueden hacer que ciertos sectores alcancen rápidamente el límite de su capacidad y se produzcan puntos de estrangulamiento por parte de la oferta, mientras que otros efectos pueden estimular la demanda en sectores con capacidad para ampliar la oferta, pero con escasa competitividad externa. En los dos casos, las importaciones (de bienes de capital, intermedios o de consumo) se incentivarán significativamente, lo que generará desequilibrios en la balanza de pagos y, posiblemente, inflación (Tavares, 1979; Furtado, 1976). En ese caso, el proceso de desarrollo solo podrá mantenerse en condiciones favorables de financiamiento externo.

vi) La falta de completitud del mercado financiero: cuando se considera el financiamiento de una empresa en particular, se supone -implícita o explícitamente- la existencia de una infraestructura financiera diversificada, que permite a la empresa evaluar las posibilidades del mercado y seleccionar la combinación entre capital propio y de terceros que más le convenga. Es decir, se supone la existencia de mercados completos. Según el enfoque teórico adoptado en este estudio, como ya se ha mostrado, esa condición no está garantizada para una empresa tomada aisladamente y aún menos para el conjunto de la economía.

Teniendo en cuenta estas condiciones, queda claro que, a diferencia de lo que proponen los enfoques de tipo tradicional, el financiamiento del desarrollo económico requiere mucho más que mercados libres y conocimientos empresariales especializados para la definición de estructuras de capital "óptimas". En ausencia de un sistema financiero diversificado y, en alguna medida, 
orientado a satisfacer las necesidades específicas de ese proceso, incluso los proyectos más prometedores podrían no ser viables debido a dificultades de financiamiento.

\section{Características específicas de los países en desarrollo}

El esfuerzo de comprensión de la heterogeneidad del desarrollo económico en los distintos países dio origen a una taxonomía en que se distingue su posición relativa en la economía internacional, utilizando tres grandes categorías:

i) los países desarrollados, en la parte superior de la clasificación, también conocidos como países industrializados, debido al predominio del sector industrial en este proceso hasta finales del siglo XX;

ii) los países subdesarrollados, expresión que fue cayendo gradualmente en desuso hasta que se sustituyó, a partir de los años ochenta, por países en desarrollo o países menos desarrollados, estratificados también por niveles de renta (media y baja), y

iii) las economías emergentes, es decir, los países en desarrollo que implementaron políticas de liberalización comercial y financiera a partir de los años ochenta y noventa, y consiguieron aumentar significativamente el peso de las relaciones internacionales en su perfil de desarrollo.

El primer indicador que suele sugerirse como distintivo de los países en desarrollo es el nivel del PIB y de sus ingresos per cápita, que históricamente ha sido inferior a los encontrados en los países industrializados con territorios y población semejantes. Estrictamente hablando, lo que distingue a los países en desarrollo en este aspecto es la persistencia de esa posición durante décadas o incluso siglos. A pesar de eso, estos países no se caracterizan exactamente por sus dificultades de crecimiento económico, aunque ese crecimiento sea un problema recurrente en muchos de ellos. La mayor dificultad reside en crear desarrollo, es decir, en combinar el crecimiento con cambios estructurales que mejoren los indicadores económicos y sociales, así como la posición del país en el mercado internacional.

"Las economías subdesarrolladas pueden conocer fases prolongadas de crecimiento de su producto global y per cápita, sin reducir el grado de dependencia externa y la heterogeneidad estructural, que son sus características esenciales" (Furtado, 1976).

Más que el bajo nivel relativo de ingresos, lo que distingue a los países en desarrollo es su dificultad para implementar $\mathrm{y}$, sobre todo, generar innovaciones en un mercado internacional ya dominado por países tecnológicamente más avanzados. En consecuencia, el retardo tecnológico constituye, para muchos autores, el principal rasgo distintivo de los países en desarrollo. A pesar de las dificultades de medición del atraso, este puede percibirse claramente a través de algunos de sus efectos: i) una baja productividad del capital y del trabajo (en contraste con los países desarrollados); ii) una dificultad crónica para competir en los mercados nacional y externo, incluso en condiciones cambiarias favorables; iii) pautas de importación y exportación concentradas, respectivamente, en bienes industriales y primarios; iv) déficits comerciales frecuentes, y v) un elevado endeudamiento externo, entre otros.

Otras condiciones distintivas de los países en desarrollo son la elevada falta de completitud del sistema financiero y de restricción externa (condiciones que también están presentes, en alguna medida, en los países industrializados $)^{3}$. Las características específicas de los países en desarrollo en estos aspectos son la mayor intensidad con que se manifiestan los problemas y la magnitud de las limitaciones que estos obstáculos imponen al proceso de desarrollo.

Según el enfoque keynesiano, como ya se ha mostrado, el sistema financiero tiende a actuar de manera poco funcional con respecto al desarrollo económico en cualquier país. El desenvolvimiento del mercado de capitales, por ejemplo, es ampliamente reconocido como un proceso difícil en cualquier país debido a los mayores riesgos y costos que supone, si se compara con el sector bancario (Herring y Chatusripitak, 2000). También en los países industrializados hay dificultades de financiamiento e incluso exclusión financiera en los segmentos de mayor riesgo (Dymski, 2005 y 2006). No obstante, en los países en desarrollo, los niveles más bajos de ingresos y las dificultades de crecimiento económico limitan la expansión del sistema financiero incluso en los segmentos de menor riesgo, haciendo que también el crédito a corto plazo resulte escaso y costoso. Por otra

\footnotetext{
${ }^{3}$ Suelen señalarse otras condiciones, no directamente relacionadas con el financiamiento del desarrollo, como causas o manifestaciones del atraso relativo de los países en desarrollo. Entre estas condiciones se encuentran: i) el elevado peso relativo de bienes de escaso valor agregado en el PIB, que refleja el menor grado de acceso del país a tecnologías más elaboradas; ii) la precariedad de la infraestructura productiva y urbana, así como de los servicios de educación y salud, realimentando el atraso tecnológico; iii) los bajos salarios, que reflejan la precaria formación de la mano de obra y la reducida tasa de inversión; iv) la mayor propensión a la inflación, tanto en las fases de crecimiento como de recesión, alimentada por diversos factores, como la alta tendencia a consumir, típica de las economías con bajos ingresos; v) la escasa productividad; vi) los conflictos de distribución, y vii) las frecuentes depreciaciones cambiarias para afrontar las restricciones externas.
} 
parte, en un país menos desarrollado es mayor el peso relativo de los grupos con un nivel más alto de riesgo y se agrava la escasez de recursos que afecta a esos grupos.

En esas condiciones, las curvas de rendimiento en los países en desarrollo asumen un perfil bastante desfavorable para los actores que necesitan fondos (Hermann, 2011b): se trata de curvas de base elevada (que reflejan el alto costo del crédito a corto plazo), de corta extensión (resultante de la falta de completitud del mercado en los segmentos a largo plazo) y con gran pendiente (que refleja el rechazo crónico de las operaciones a largo plazo por parte del sistema financiero y que se manifiesta también en el elevado costo de las operaciones que escapan al racionamiento del crédito) (véase el gráfico 1).

En cuanto a la restricción externa, ante la creciente relevancia de las relaciones comerciales y financieras internacionales, el proceso de desarrollo de cualquier país está condicionado - y eventualmente limitadopor el desempeño de la balanza de pagos. En los países en desarrollo, sin embargo, esa restricción suele tomar
GRÁFICO 1

Países desarrollados y en desarrollo: condiciones de financiamiento

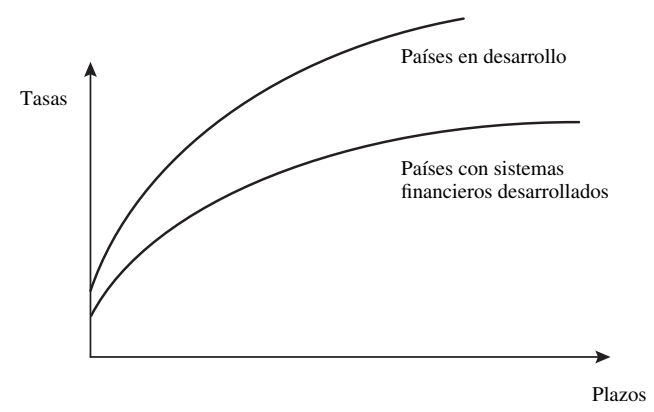

Fuente: elaboración propia.

la forma de un problema crónico, porque deriva de una condición estructural: la mayor dependencia de esos países con respecto al financiamiento externo, ya sea para atenuar la falta de completitud del sistema financiero o para cubrir los desequilibrios de la balanza de pagos, asociados al atraso tecnológico.

\section{IV}

\section{Funcionalidad del sistema financiero para el desarrollo económico}

Una condición básica para que el sistema financiero actúe de manera funcional para el desarrollo económico en cualquier país, es que se disponga de un conjunto diversificado de instituciones e instrumentos financieros capaces de satisfacer las variadas demandas vinculadas a ese proceso, así como de los objetivos de lucro de las propias instituciones financieras (Carvalho, 2010). Empero, esa condición no es equivalente de los conceptos de eficiencia y desarrollo financiero, en los sentidos más usuales de dichos términos. En el primero, común a los enfoques de filiación neoclásica, se subrayan las funciones microeconómicas del sistema financiero, es decir, aquellas destinadas a satisfacer los objetivos de lucro de las instituciones financieras y de valorización de la riqueza de sus clientes. En ese sentido, la hipótesis de mercados eficientes asegura que la eficiencia en estas funciones es condición necesaria y suficiente para que el sistema financiero cumpla también su función macroeconómica de proveer financiamiento para las actividades productivas.
En el enfoque keynesiano no se niega la importancia de esas funciones microeconómicas, pero se rechaza la hipótesis de los mercados eficientes y, con ella, la equivalencia de eficiencia micro y macroeconómica del sistema financiero. Además de la tendencia natural de los sistemas financieros a la falta de completitud con respecto a las necesidades del desarrollo económico, se da el hecho de que las condiciones que definen la eficiencia del sistema financiero no se cumplen necesariamente en bloque. Un sistema financiero puede llevar a cabo la función de financiamiento con una estructura poco diversificada - concentrada en bancos comerciales, por ejemplo- y poco eficiente en cuanto a la valorización de la riqueza. Esta fue la contradicción señalada en los países en desarrollo por los teóricos de la liberalización financiera (Shaw, 1973; McKinnon, 1973), que atribuyeron el problema a la política de represión financiera, es decir, a la fuerte actuación del Estado a través de la reglamentación y los programas de crédito público. 
También es posible que un sistema financiero logre el objetivo de valorización de la riqueza, pero no cumpla adecuadamente la función de financiamiento. Este ha sido el caso de muchos países en desarrollo después de las políticas de liberalización de los años noventa, que promovieron la expansión y diversificación de los sistemas financieros, manteniendo no obstante gran parte de las dificultades de financiamiento existentes, entre ellas la exclusión financiera y la dependencia externa (Hermann, 2004).

La funcionalidad del sistema financiero tampoco es equivalente a la noción de su desarrollo cuando esta asume el sentido puramente cuantitativo y más usual del término. Así, el grado de desarrollo se mide mediante indicadores que se agregan, como el número y los tipos de instituciones financieras, el valor agregado de sus activos, la participación relativa del sector y del crédito en el PIB, entre otros ${ }^{4}$. Aunque sean relevantes, esos indicadores resultan insuficientes para captar el concepto más amplio de funcionalidad macroeconómica. A partir de los enfoques de Keynes y Minsky, además de la existencia física de una estructura financiera diversificada, es necesario que un sistema financiero funcional para el desarrollo económico sea capaz de cumplir dos condiciones esenciales: i) la satisfacción de la demanda de fondos en sus diversas modalidades, teniendo en cuenta las necesidades de financiamiento iniciales y de largo plazo, y ii) el control del nivel de fragilidad financiera, ya que es inevitable que exista un cierto grado de fragilidad:

"La funcionalidad se define como sigue: un sistema financiero es funcional para el proceso de desarrollo económico cuando expande el uso de los recursos existentes en el proceso de desarrollo económico, con el mínimo incremento posible de la fragilidad financiera y otros desequilibrios, que podrían detener el proceso de crecimiento por razones puramente financieras" (Studart, 1995, pág. 64).

Las condiciones específicas de los países en desarrollo sugieren dos ampliaciones del concepto de Studart. En primer lugar, la expansión de los recursos existentes debe cumplir ciertos criterios de asignación para reducir el grado de racionamiento del crédito y la exclusión financiera que afecta a sectores esenciales para el desarrollo. Un sistema financiero funcional para el desarrollo económico en dichos países debe disponer de variados mecanismos de financiamiento para apoyar: i) las inversiones en innovaciones, en todas sus fases;

4 Un ejemplo de este tipo de enfoque puede encontrarse en BID (2005, cap. 1). ii) la ampliación y modernización de la infraestructura productiva y urbana; iii) a las pequeñas y medianas empresas (pymes); iv) a los sectores con características de bienes públicos; v) el financiamiento de la vivienda, y vi) el financiamiento del consumo de bienes duraderos (teniendo en cuenta también, en los dos últimos casos, a la población con bajo nivel de ingresos).

En cuanto al financiamiento de innovaciones, ante el elevado grado de incertidumbre y el racionamiento del crédito, la experiencia de países más desarrollados apunta al mercado de capitales como el canal más adecuado, fundamentalmente a través de fondos de participación en nuevas empresas (capital de riesgo). De esta forma, el cumplimiento del requisito de funcionalidad supone una exigencia más específica con respecto a la estructura del sistema financiero: el desarrollo del mercado de capitales.

La segunda ampliación necesaria se refiere a la fragilidad financiera: un sistema financiero funcional para el desarrollo económico en los países en desarrollo debe contar (en el ámbito de lo que el autor denomina "otros desequilibrios") con mecanismos de control de la fragilidad financiera externa. Por analogía con el concepto original de Minsky, dicha fragilidad está determinada por el desajuste de las monedas, que puede deberse a la acumulación de deuda en divisas por actores con ingresos en la moneda nacional (es decir, todos, excepto los exportadores), o al desajuste de plazos en las deudas externas de los exportadores.

Por lo tanto, según la perspectiva teórica keynesiana, el concepto de funcionalidad debe tener en cuenta cuatro aspectos: i) el volumen de recursos; ii) la asignación a sectores estratégicos para el desarrollo; iii) la fragilidad financiera interna, y iv) la fragilidad financiera externa. Aunque, desde el punto de vista analítico, esos aspectos se traten separadamente y en general requieran diferentes instrumentos de promoción, no son independientes entre sí, porque la ausencia de alguno de ellos puede dificultar el cumplimiento de los demás. Por ejemplo, una expansión excesiva del crédito y una asignación inadecuada de los recursos (con plazos inferiores al ideal o costos muy altos) incrementan el grado de fragilidad financiera de la economía. Esta situación podría extenderse a la fragilidad externa, si esa inadecuación del sistema financiero fuera compensada por un elevado endeudamiento externo. Finalmente, un alto grado de fragilidad financiera o de fragilidad externa de la economía tiende a inhibir la oferta de fondos, lo que dificulta el cumplimento de la condición relativa al volumen de recursos.

Los ámbitos de la asignación y de la fragilidad externa son especialmente significativos en los países en desarrollo. Ante la dificultad crónica para obtener 
financiamiento en el mercado interno, la fragilidad externa se convierte en una tendencia casi inevitable en las fases de amplia liquidez internacional. Salvo en presencia de obstáculos al capital extranjero (económicos o de otro tipo), esas fases provocan una pronunciada expansión del endeudamiento externo en los países en desarrollo. De esa forma, parte de la fragilidad financiera de esos países se manifiesta como fragilidad externa.

Con esta interpretación no se pretende sugerir que el volumen de recursos sea irrelevante, sino solo resaltar que, aisladamente, ese aspecto proporciona poca información sobre el grado de funcionalidad del sistema financiero en los países en desarrollo. Supuestamente, el aumento del volumen de recursos constituye un indicador del progreso de las condiciones de financiamiento en cualquier país, pero esa hipótesis solo se confirma si ese incremento está adecuadamente dirigido, reduciendo el grado de racionamiento de crédito y de exclusión financiera, sin acrecentar excesivamente las fragilidades financiera y externa.

\section{V}

\section{Políticas para aumentar la funcionalidad de los sistemas financieros en los países en desarrollo}

Una importante consecuencia práctica de la teoría keynesiana de finanzas es que, ante la tendencia natural del sistema financiero a la falta de completitud y a la fragilidad financiera, es poco probable que el sistema cumpla las condiciones de funcionalidad para el desarrollo económico en ausencia de la actuación del Estado en este sentido (Carvalho, 2010; Hermann, 2011a) a través de: i) políticas que incentiven el desarrollo del sistema financiero y procuren reducir su falta de completitud; ii) políticas de control de la fragilidad de las finanzas interna y externa, teniendo siempre en cuenta que esas tendencias no pueden eliminarse totalmente del sistema financiero, y iii) políticas compensatorias que complementen las otras dos. En esta sección se discuten posibles líneas de actuación en cada una de esas áreas, considerando las cuatro dimensiones antes mencionadas del concepto de funcionalidad financiera para los países en desarrollo.

\section{Políticas relativas al volumen y la asignación de recursos}

La política necesaria para fomentar que el sistema financiero incremente su actuación en los segmentos esenciales para el desarrollo económico debe definirse de acuerdo con el grado y el perfil de falta de completitud del sistema en cada país en desarrollo. A pesar de sus características específicas, las dificultades de financiamiento de estos países tienen rasgos comunes: la escasez y el alto costo de las fuentes privadas de financiamiento, tanto a corto como a largo plazo, sintetizados en curvas de rendimiento altas, cortas e inclinadas.
Para que esa situación mejore se requiere que, en el ámbito de las políticas de incentivos, se tomen medidas de estímulo de la demanda de activos financieros, con especial atención a los de largo plazo -incluidos los de renta variable (acciones) — del mercado de capitales. En el campo de las políticas compensatorias, se recomiendan programas de direccionamiento del crédito privado $\mathrm{y}$, sobre todo, políticas de crédito público dirigidas a los sectores esenciales para el desarrollo en cada período, con condiciones de plazos y costos más accesibles que las (que estarían) presentes en el mercado (en los segmentos en que exista tal opción).

Como se argumenta a continuación, estas políticas en conjunto promueven el rebajamiento, el alargamiento y la reducción de la inclinación de las curvas de rendimiento de los diversos segmentos del sistema financiero. Además, contribuyen indirectamente a disminuir el grado de fragilidad financiera vinculado al proceso de desarrollo económico, porque las mejores condiciones de plazos y costos reducen el riesgo de crédito.

La demanda de un determinado activo está condicionada por las estimaciones de retorno y riesgo del propio activo frente a los activos alternativos; por el entorno macroeconómico, que aumenta la preferencia por la liquidez en escenarios de gran incertidumbre; por títulos a más largo plazo en escenarios de mayor optimismo, y por la estructura de incentivos marcada por la política financiera (Carvalho, 2010). Además de un ambiente macroeconómico con baja incertidumbre, el estímulo de la demanda de activos a largo plazo requiere una estructura de incentivos que establezca alguna ventaja 
comparativa de esos activos con respecto a los activos de menor riesgo. Esta estructura puede crearse de dos formas, que no son mutuamente excluyentes: mediante el aumento de las tasas de retorno (ajustadas al riesgo) de los títulos a largo plazo o mediante la reducción de las tasas de interés de los títulos a corto plazo. El perfil de la curva de rendimientos en los países en desarrollo indica que la segunda forma es la más recomendable.

La reducción de los intereses a corto plazo favorece las operaciones a largo plazo (incluido el mercado de capitales) de dos maneras: i) a través del rebajamiento de la curva de rendimientos, que reduce los costos de todas las operaciones financieras -incluidas las operaciones a largo plazo - al promover el alargamiento de la curva, y ii) mediante la probable disminución de la inclinación de la curva de rendimientos, que refuerza el efecto anterior, ya que ante un menor retorno de los títulos a corto plazo, suele incrementarse la preferencia de los inversionistas por activos a más largo plazo que, en el nuevo escenario (con bajos intereses), se convierten en el único medio de elevar la rentabilidad de su cartera (véase el gráfico 2).

GRÁFICO 2

Países en desarrollo: efectos de la reducción de los intereses a corto plazo en la curva de rendimiento

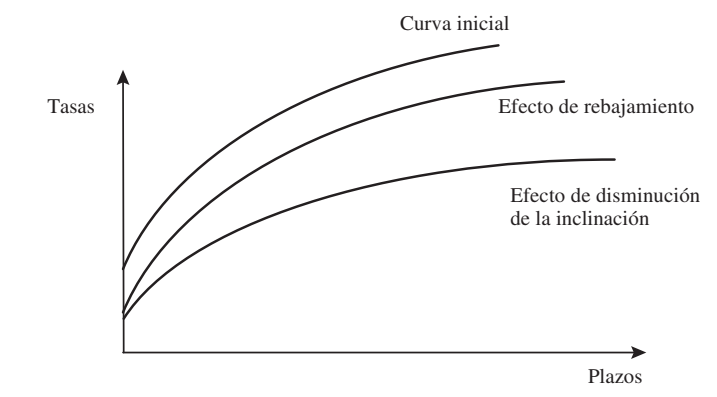

Fuente: elaboración propia.

Una política sistemática que promueva los bajos intereses a corto plazo contribuye también a un ambiente macroeconómico más favorable al desarrollo financiero. Las tasas de interés elevadas tienden a influir de forma perversa en la demanda de activos en general, debido al efecto de selección adversa que eleva el riesgo de crédito para los acreedores (Stiglitz y Weiss, 1981) y, posiblemente, pone en peligro su rentabilidad. En ese contexto, crece el grado de incertidumbre y la preferencia por la liquidez, de manera que se penalizan especialmente los activos a plazos más largos.

Unas tasas de interés a corto plazo adecuadamente bajas, es decir, que no den lugar a los desequilibrios macroeconómicos mencionados a continuación, tienden a reducir los riesgos del endeudamiento y, al estimular el crecimiento económico, mejoran las expectativas de retorno de los activos, incluidos los activos a largo plazo. Los límites de esa política son, naturalmente, los riesgos de inflación, de sobreendeudamiento (y, por lo tanto, de crisis financiera) y de desequilibrios en la balanza de pagos. No obstante, incluso frente a esos riesgos, los efectos perversos de la política de tasas de interés altas implican que se trata de una opción que debe utilizarse con cautela, de manera temporal y complementándola con otros instrumentos de actuación más directa sobre esos desequilibrios macroeconómicos (cuya discusión escapa a los objetivos de este artículo).

En resumen, en este trabajo se rechaza la idea, defendida en el modelo Shaw-McKinnon y ampliamente difundida en este debate, de que tasas de interés elevadas puedan contribuir al desenvolvimiento económico, ya sea en los países en desarrollo o desarrollados, ampliando la oferta de fondos para préstamos. Según el enfoque keynesiano, tasas de interés más altas pueden, al menos como hipótesis, elevar la rentabilidad de las instituciones financieras, estimulando el crédito. Esta posibilidad, sin embargo, está sujeta a dos tipos de riesgo: aquel asociado al rápido aumento de la morosidad de los deudores y el consiguiente evento de que se produzca una crisis financiera. En los dos casos, la rentabilidad de las instituciones financieras se verá afectada negativamente y se interrumpirá la expansión del crédito. Así pues, se entiende que una política de bajas tasas de interés a corto plazo conllevaría más beneficios que daños para el proceso de desarrollo económico: sumadas a los diferenciales de riesgo de cada modalidad de operación financiera (reducidos, idealmente, por la política macroeconómica), esas tasas de interés darían lugar a tasas finales razonables para los tomadores de crédito y compatibles con niveles de rentabilidad también razonables y satisfactorios para las instituciones financieras. Por último, la política de crédito dirigido requiere, en primer lugar, la creación de fuentes seguras de financiamiento a largo plazo, a fin de evitar que los bancos, privados o públicos, que asuman esta función se conviertan en fuentes de incremento de la fragilidad financiera (y también fiscal, en el caso de los bancos públicos) que deberían mitigar. Ante el entorno de restricción de la demanda de activos a largo plazo en que deben implementarse esas políticas, la fuente de financiamiento a largo plazo recomendable para apoyarlas es la de recursos exteriores al mercado, es decir, de origen fiscal o parafiscal (ahorro obligatorio). 
En segundo lugar, para que esas políticas cumplan, de hecho, su función de compensar la falta de crédito destinado a los sectores considerados como estratégicos para el desarrollo, es necesario asegurar que ese crédito efectivamente se conceda cuando se solicite. En ese sentido, el crédito público tiende a ser más eficaz que el crédito dirigido. Este último, aunque pueda contribuir a reducir la falta de completitud del mercado, sufre una limitación común a las formas más indirectas de actuación del Estado en el sistema financiero (supervisión, reglamentación e incentivos): la de ser una política no coercitiva, que solo actúa mediante incentivos.

Los programas de crédito dirigido crean reglas de asignación a partir de determinadas fuentes privadas de captación, o proporcionan fondos públicos (fiscales o parafiscales) con costos inferiores a los de mercado, de modo que esas instituciones concedan los créditos en cuestión. Con esas medidas se procura fomentar que las instituciones privadas concedan el crédito, pero no las obligan a hacerlo, de modo que su eficacia puede estar limitada por la misma falta de interés que causa la falta de completitud del mercado. Así, la única forma garantizada de atenuar esa situación consiste en que el gobierno asuma directamente los riesgos que rechaza el sector privado. Esto requiere la creación de bancos de desarrollo, es decir, bancos públicos con funciones específicas en el proceso de desenvolvimiento económico.

\section{Políticas relativas a la fragilidad financiera}

\section{a) Fragilidad financiera interna}

El control de la fragilidad financiera puede apoyarse, en parte, en mecanismos de cobertura de mercado, como activos de alta liquidez, mercados secundarios organizados, formadores de mercados, mercados futuros o instrumentos derivativos, entre otros. Sin embargo, para que se produzcan estas operaciones es necesario que el sistema financiero cuente con un mercado de capitales razonablemente desarrollado, algo que raramente sucede en los países en desarrollo. Esa limitación refuerza la necesidad, anteriormente señalada, de coordinación entre las políticas de asignación y de control de la fragilidad financiera en los países en desarrollo.

No obstante, incluso en los países que cumplan en alguna medida esa condición, teniendo en cuenta que las operaciones de cobertura se basan también en las expectativas (al igual que las operaciones protegidas por ellas), esos instrumentos de mercado pueden resultar insuficientes o incluso contraproducentes como medio de defensa del sistema financiero. Así sucede, por ejemplo, en escenarios de mayor volatilidad de los precios de los activos, que fomentan las operaciones de carácter especulativo (destinadas a obtener lucro y no exactamente a la protección), así como las motivadas por el comportamiento gregario, ya que la volatilidad dificulta la realización de estimaciones fiables (Kregel, 1997).

En esas condiciones, la negociación de esos instrumentos sobre una base relativamente segura requiere, además de una infraestructura física (de instituciones financieras y mercados), una infraestructura institucional (reglamentaria) que actúe en dos frentes: i) en el control preventivo de los riesgos asumidos en el sistema financiero, y ii) en la compensación de sus efectos macroeconómicos cuando las medidas preventivas se muestren insuficientes o ineficaces.

Una normativa preventiva debe centrarse en la protección del ahorrador final y en el control de la fragilidad financiera. Lo primero requiere, básicamente, una reglamentación que establezca la responsabilidad de las instituciones financieras respecto de las informaciones relativas a los riesgos de las operaciones originadas a partir de la captación de recursos de los ahorradores y, al menos en parte, de las posibles pérdidas resultantes. Además de la protección del ahorrador, de por sí necesaria, esa reglamentación contribuye, indirectamente, al desarrollo del sistema financiero nacional, al hacer que el mercado sea más atractivo para los ahorradores en general.

En cuanto a la fragilidad financiera, aunque la reglamentación prudencial solo pueda versar sobre operaciones específicas, su objetivo final, desde el punto de vista de la funcionalidad del sistema financiero, debe ser el control del riesgo sistémico, es decir, del grado de exposición de la economía a los riesgos del sistema financiero. En ese aspecto, además de los riesgos asumidos o provocados por actores específicos, resultan significativas las posibles vías de contagio entre ellos. El control del riesgo sistémico requiere la imposición de restricciones a la acumulación de deudas especulativas (incluidos los esquemas Ponzi) — como medio de control de los riesgos asumidos- $y$ al grado de apalancamiento de las instituciones financieras para limitar el contagio entre ellas.

No obstante, el perfil de endeudamiento de los actores y las vías de contagio en el sistema financiero no son circunstancias claramente perceptibles en la economía. Así, a pesar del mayor costo macroeconómico que supone restringir el potencial de expansión de todo el mercado, la actuación para prevenir el riesgo sistémico debe apoyarse, esencialmente, en el control del nivel general de exposición del sistema financiero a los riesgos. Históricamente, ese control se ha llevado a cabo por medio de la imposición de barreras reglamentarias y 
de costos a las instituciones financieras, asociados a la expansión de sus operaciones activas (Carvalho, 2005).

Las barreras reglamentarias suelen tomar la forma de segmentación del mercado, vetando la actuación de bancos comerciales en el mercado de capitales, según el modelo de la Ley Glass-Steagall (1935) de los Estados Unidos de América. El objetivo principal de ese veto es evitar el contagio entre los dos segmentos del sistema financiero. Aunque se utilizó con éxito en los Estados Unidos de América y en diversos otros países hasta la década de 1980, ese modelo cayó en desuso a partir del decenio de 1990 con la difusión de las políticas de liberalización (también iniciadas en los Estados Unidos de América), que promovieron una eliminación gradual de barreras reglamentarias a la libre elección de su cartera por parte de las instituciones financieras.

En ese nuevo modelo de sistema financiero queda, como única alternativa, la imposición de costos a las opciones con mayor potencial para provocar un riesgo sistémico, esencialmente las que implican un mayor riesgo de liquidez (Carvalho, 2009). Esos costos han asumido diversas formas (Carvalho, 2005): exigencia de niveles mínimos de liquidez y de capital en el balance de las instituciones financieras (recaudaciones obligatorias sobre depósitos, provisiones de crédito y regla de Basilea, entre otros); reglamentación del mercado de derivados (operaciones fuera del balance) en la misma línea (exigencia de márgenes de liquidez y capital), y medidas tributarias, entre otras.

En los países en desarrollo clasificados como mercados emergentes, donde el mercado de capitales está razonablemente desarrollado, la reglamentación prudencial debe extenderse a este mercado, en sus dos segmentos: el de deuda corporativa y el de acciones. El primero se asemeja al crédito bancario, excepto por el mayor grado de liquidez de los títulos corporativos, aunque normalmente tampoco sea muy alto (Herring y Chatusripitak, 2000). Las acciones no contribuyen a elevar el nivel de endeudamiento de la economía, sino que, al contrario, constituyen una alternativa a la deuda, pero tampoco están exentas de riesgo sistémico. Este riesgo se deriva sobre todo: i) del riesgo de mercado, típico de las acciones; ii) de la mayor exposición de ese segmento al comportamiento gregario, facilitado por la mayor liquidez de las acciones en comparación con otros tipos de activos, y iii) del hecho de que muchos inversionistas, especialmente los de mayor tamaño, actúan apalancados, es decir, aplican recursos obtenidos mediante préstamos, provocando un riesgo de contagio del sector bancario en caso de un desempeño desfavorable del mercado de acciones.
El control del riesgo sistémico asociado al mercado de capitales requiere, pues, instrumentos similares a los aplicables a los bancos: obligación de que las instituciones intermediarias se responsabilicen de la correcta divulgación de la información y de las posibles pérdidas resultantes de fallos en este aspecto; exigencia de márgenes de liquidez y capital, y límites a la acumulación de títulos en los balances y al grado de apalancamiento de las instituciones financieras.

La eficacia del modelo de control de riesgo basado en costos está, sin embargo, restringida por factores en gran parte ajenos a las autoridades reguladoras, como por ejemplo, la fase del ciclo económico y el continuo proceso de innovación financiera. En lo tocante al ciclo económico, se puede esperar menor eficacia de la reglamentación prudencial en las fases expansivas y de mayor optimismo del mercado, justamente cuando el control de la expansión financiera es más necesario. En ese escenario, el sistema financiero tiende a minimizar el peso de los costos reglamentarios ante la expectativa de compensarlos más fácilmente con los mayores ingresos previstos. Esa limitación puede atenuarse (aunque no superarse totalmente) mediante una estructura anticíclica de costos reglamentarios basada, por ejemplo, en una escala progresiva de costos según el volumen de recursos o según la tasa de expansión de cada tipo de operación que se desee controlar.

Las innovaciones financieras limitan doblemente la eficacia de los controles preventivos de la fragilidad financiera a través de la imposición de costos. Por una parte, hacen que la estructura de costos vigente se vuelva gradualmente obsoleta, a medida que algunos tipos de transacción pierden importancia relativa en el mercado. Por otra, dificultan la reformulación de los instrumentos de control en la medida en que los nuevos tipos de transacción aún son poco conocidos:

"La innovación (tanto financiera como organizativa) [...] es en sí misma una fuente de inadecuación institucional. [...] [P]or el efecto del desarrollo de los sistemas financieros, las instituciones formales [...] pierden eficacia y dejan de contrarrestar suficientemente la dinámica endógena de asunción de riesgos" (Sinapi, 2011, pág. 18).

La existencia de esas limitaciones indica la conveniencia de recurrir a políticas compensatorias de intervención más directa en el sistema financiero complementarias de la reglamentación prudencial, tales como: i) barreras reglamentarias para la asunción de riesgos por parte de las instituciones financieras; ii) instrumentos de cobertura de emergencia en momentos de mayor incertidumbre (por ejemplo, operaciones de 
mercado abierto de emergencia con títulos públicos y divisas); iii) préstamos de última instancia del banco central a instituciones financieras con dificultades temporales de liquidez; iv) programas de reestructuración del sector bancario ante dificultades prolongadas que denoten problemas de solvencia, y v) mecanismos de circuito abierto en el mercado de capitales (implementados por reguladores oficiales o por órganos privados de autorregulación), entre otros.

\section{b) Fragilidad financiera externa}

La fragilidad financiera externa de los países en desarrollo, como ya se ha indicado, tiene raíces estructurales, vinculadas al perfil de su estructura productiva y a la consiguiente posición más frágil de esos países en el mercado financiero internacional. Esas condiciones escapan del ámbito de actuación de la política financiera, pero esta tiene un papel significativo en el control del riesgo sistémico asociado al posible contagio del sistema financiero doméstico, en la medida en que la fragilidad externa del país dé lugar a dificultades de liquidez para las instituciones financieras. Ese contagio puede producirse por tres vías: a través de las obligaciones externas de las propias instituciones financieras y del sector no financiero (incluidas, en ambos casos, las operaciones de balance y fuera de balance) y a través del mercado de divisas.

La primera vía provoca, en particular, el contagio del sistema financiero a la balanza de pagos, ya que en este caso el pasivo del sistema financiero constituye una de las causas de la fragilidad externa del país. El contagio a través de la segunda vía se produce como contrapartida de la fragilidad externa del sector no financiero, cuya demanda de divisas puede desencadenar un movimiento masivo de cancelación de las inversiones financieras internas, elevando el pasivo a cortísimo plazo (en reservas) de los bancos. La aparición de crisis sistémicas a través de esas dos vías se puede evitar en la medida en que el flujo de obligaciones externas sea compatible con la disponibilidad de divisas en el país. De esta forma, el riesgo sistémico asociado a esas vías puede contenerse con una estrategia semejante a la señalada anteriormente para el control de la fragilidad financiera interna: la imposición de límites reglamentarios a la acumulación de obligaciones externas por parte del sistema financiero y del sector no financiero, unida a la diferenciación de las medidas de control que deben focalizarse en las transacciones a corto plazo, que mantienen sistemáticamente elevada la demanda de divisas.

El contagio a través del mercado de divisas se produce por un comportamiento adverso del tipo de cambio, capaz de ocasionar pérdidas significativas al sistema financiero. Teniendo en cuenta la mayor propensión de los países en desarrollo a la fragilidad externa, una depreciación del tipo de cambio es particularmente arriesgada, porque eleva el costo de las obligaciones externas del país, incluidas las del sistema financiero, y actúa como agravante de las dos vías anteriores. Los movimientos de apreciación del tipo de cambio, sin embargo, no son necesariamente beneficiosos para el sistema financiero de los países en desarrollo. La apreciación tiende a empeorar la posición del país en el comercio internacional y a agravar, por tanto, la situación de fragilidad externa. Por otra parte, en los países en desarrollo que cuentan con mercados de futuros razonablemente desarrollados (la mayoría de los mercados emergentes), tanto la depreciación como la apreciación del tipo de cambio pueden generar grandes pérdidas para el sistema financiero, en caso de que el sistema esté ampliamente comprometido con derivados cambiarios: basta que la dirección o la intensidad del movimiento cambiario sorprendan a gran parte del mercado.

En ese contexto, además de la ya mencionada imposición de límites a la asunción de obligaciones externas, es necesario implementar una política permanente de control de la volatilidad cambiaria. La primera reduce el grado de exposición del sistema financiero al riesgo cambiario y la segunda, al garantizar una mayor previsibilidad del tipo de cambio, tiende a disminuir el propio riesgo cambiario correspondiente a cada tipo de operación que dé lugar a obligaciones externas.

En los regímenes de tipo de cambio administrado, que predominaron en los países en desarrollo hasta finales de la década de 1990, la volatilidad cambiaria se evitaba mediante la actuación constante del banco central, que se comprometía a mantener el tipo de cambio en el nivel establecido. Como señalan los modelos de segunda generación (Obstfeld, 1994), la sostenibilidad de esos regímenes está condicionada por la confianza del mercado y especialmente de las instituciones financieras en la capacidad del banco central de cumplir el objetivo cambiario anunciado. Si esa condición no se cumple, el país se vuelve vulnerable a ataques especulativos (como un súbito aumento de la preferencia por divisas, en detrimento de las inversiones en moneda nacional), capaces de obligar al banco central a alterar el objetivo cambiario o, en casos extremos, a abandonar el régimen de tipo de cambio administrado. La grave conmoción que sufrió la credibilidad de esos regímenes, impulsada por las políticas de liberalización comercial y financiera implementadas en los países en desarrollo en los años noventa, como se sabe, desencadenó una serie de crisis cambiarias en esos países y provocó la 
sustitución del antiguo modelo por el régimen de tipo de cambio flotante.

En los regímenes de tipo de cambio flotante la volatilidad cambiaria es parte del juego, ya que, por definición, el banco central no asume un compromiso formal con respecto a la tasa de cambio. Por otra parte, el incuestionable compromiso de cualquier gobierno con la estabilidad macroeconómica, que requiere un cierto grado de estabilidad monetaria y del sistema financiero, exige que, en regímenes de tipo combinado de cambio flexible, los bancos centrales asuman la función de contener la volatilidad cambiaria. Esto dio origen a los regímenes de flotación sucia, que predominaron a partir de finales de los años noventa.

En ese modelo, el control de la volatilidad cambiaria puede realizarse a través de actuaciones del banco central en los mercados a la vista y de futuros, negociando divisas y derivados cambiarios. En las operaciones a la vista, el banco actúa directamente sobre el tipo de cambio corriente y, en el mercado de futuros, influye en las expectativas cambiarias. En ambos casos, sin embargo, el banco central no es totalmente inmune a las dificultades resultantes de la alta movilidad de capital, que limitaban su capacidad de controlar el tipo de cambio en los regímenes de tipo de cambio administrado. Concretamente, en escenarios de gran incertidumbre, el comportamiento gregario del sistema financiero limita la capacidad de

\section{VI}

\section{Conclusiones}

Un sistema financiero funcional para el desarrollo económico debe ser capaz de satisfacer sus necesidades de financiamiento con condiciones adecuadas de plazos y costos. La ausencia de esas condiciones no impide, necesariamente, el avance de este proceso, pero le impone una dinámica de tipo intermitente, con frenazos y aceleraciones, fruto de una estructura de financiamiento con un elevado grado de fragilidad financiera (ya que está concentrada en deudas a corto plazo y con alto costo) y también, posiblemente, de fragilidad externa en los casos en que la inadecuación del sistema financiero nacional pueda verse atenuada por el endeudamiento externo. De esta forma, la funcionalidad del sistema financiero no es solo una condición deseable y que facilita el desarrollo económico, sino necesaria para que este proceso no se convierta en una fuente de riesgo sistémico y, probablemente, actuación del banco central ante fuertes tendencias de apreciación o depreciación del tipo de cambio. La imposibilidad de evitar definitivamente esos escenarios indica que, además de la influencia del banco en el precio de la moneda, conviene instituir mecanismos de control de los propios flujos de capital que condicionan ese precio.

El control anticíclico y selectivo de los ingresos de capital es particularmente relevante en los países en desarrollo, debido a una mayor propensión de estos al endeudamiento externo en las fases de mayor optimismo de los inversionistas extranjeros. Las barreras (normativas o tributarias) para los ingresos de capital en esas fases, con mayores restricciones de las operaciones a corto plazo, contribuyen a evitar una expansión excesiva del endeudamiento externo. Análogamente, en las fases de gran pesimismo, el control selectivo puede utilizarse para disciplinar el movimiento de salida de capital del país. En los dos casos, la fragilidad externa del país y la volatilidad del mercado cambiario se atenúan.

Cabe notar, finalmente, el riesgo de que las políticas de control de la fragilidad financiera entren en conflicto con las necesidades de financiamiento del desarrollo, porque tienden a restringir el volumen total de operaciones del sistema financiero. Ese dilema demuestra la extrema importancia de la coordinación de ese control con las políticas de asignación antes mencionadas para la formación de unas finanzas más funcionales al desarrollo.

de crisis bancarias y cambiarias que suponen un gran sacrificio para la economía.

Según la perspectiva keynesiana, como ya se ha mostrado, las condiciones normales de actuación del sistema financiero tienden a hacer que sea generalmente incompleto y disfuncional, incluso en países que se encuentran en un estado avanzado de desarrollo económico y financiero. Así pues, resulta poco plausible que se cumplan esas condiciones en ausencia de políticas de Estado dirigidas a este fin. En los países en desarrollo, las dificultades se ven agravadas por las propias características que los distinguen como menos desarrollados —en especial, la baja diversificación estructural del sistema financiero y la intermitente dependencia financiera externa-. Esas características hacen que los países en desarrollo estén más sujetos a períodos de crecimiento sin desarrollo (Furtado, 1976), así como a la fragilidad 
financiera, sobre todo en su dimensión externa, de manera que se crea un círculo vicioso que perpetúa su posición desfavorable en el ámbito internacional.

La atenuación de esa situación requiere un conjunto coordinado de políticas financieras, apoyado en dos pilares: i) las políticas de asignación, centradas en los segmentos estratégicos para el desarrollo económico y financiero, y ii) las políticas de control de la fragilidad financiera, doméstica y externa. Teniendo en cuenta, no obstante, que incluso los sistemas financieros amplios y diversificados mantienen rasgos de comportamiento disfuncionales para el desarrollo económico (como el escaso interés por proyectos con perfil de bienes públicos o la exclusión financiera, entre otros); en tal caso las políticas convencionales, basadas en incentivos y costos dirigidos a mercados desregulados, serán insuficientes para fomentar que los sistemas financieros de los países en desarrollo alcancen un grado adecuado de funcionalidad.

En efecto, también se necesitan políticas compensatorias de intervención más directa sobre lo que se pretende estimular o contener. En este grupo destacan: i) los programas de crédito dirigido, privado o público, en el campo de las políticas de asignación (que también tienen efectos beneficiosos indirectos en el control de la fragilidad financiera); ii) la imposición de barreras reglamentarias (externas al mercado) en el campo de las políticas de control de la fragilidad financiera, y iii) las medidas anticíclicas de restricción del libre flujo de capitales para el control de la fragilidad externa.

Las políticas intervencionistas tampoco están exentas de riesgos. Las dificultades que encuentran los actores en general para prever el comportamiento de indicadores relevantes también afectan a los órganos de gobierno responsables de la ejecución de esas políticas. Aun así, la historia financiera del siglo XX y las primeras décadas del presente siglo sugiere que los daños macroeconómicos de ese riesgo de previsión pueden ser menores que los de los riesgos de mercado 5 .

5 La excepción es el riesgo político (de corrupción, por ejemplo) vinculado a cualquier tipo de acción de gobierno. Sin embargo, este problema no es específico de la política financiera ni tampoco de la política económica en general, sino que es de naturaleza política y jurídica y debe tratarse en estos ámbitos.
Durante los cinco decenios de vigencia del modelo intervencionista, desde mediados de los años treinta hasta los años ochenta, ninguna crisis sistémica sacudió a la economía mundial, a pesar del indiscutible avance del proceso de desarrollo económico y financiero. En ese período, se produjeron dos crisis del crédito inmobiliario en el mundo (en los años setenta y ochenta), originadas en países ricos con gran influencia en el mercado internacional (los Estados Unidos de América y el Japón), además de la crisis de la deuda externa en América Latina durante la década de 1980. Aunque causaron graves perjuicios a los países afectados, el hecho es que, ante las barreras reglamentarias de la época, ninguna de ellas se convirtió en una crisis sistémica internacional.

Por otra parte, los resultados de los últimos años no han supuesto un gran apoyo para el modelo de política financiera convencional, basada en incentivos y predominante en la era de la liberalización. En poco más de 20 años de vigencia, este modelo no ha sido capaz de evitar dos crisis sistémicas de alcance internacional, en un contexto de menor crecimiento económico que en el período anterior: la crisis de los países en desarrollo asiáticos, que comenzó en 1997 y se extendió a los países de América Latina entre 1999 y 2003, y la crisis iniciada en 2008 (y todavía en curso en 2013), que surgió de forma significativa en los Estados Unidos de América, la "cuna de la liberalización". La segunda, al afectar más gravemente a los países ricos, con sistemas financieros desarrollados, refuerza la hipótesis de la ineficacia del modelo actual.

Incluso teniendo en cuenta las diferencias de contexto macroeconómico, que impiden una comparación lineal entre los períodos y los países correspondientes, resulta difícil no asociar esas crisis al cambio en el modelo de control del riesgo sistémico resultante de la liberalización financiera. Los eventos recientes hacen conveniente, como mínimo, que se vuelva a colocar el tema de las políticas intervencionistas (no necesariamente con las modalidades de la antigua versión) en la agenda de los debates sobre posibles formas de lograr que el sistema financiero sea más funcional para el desarrollo económico, no solo en los países en desarrollo, sino también en los países industrializados. 


\section{Bibliografía}

BID (Banco Interamericano de Desarrollo) (2005), Relatório 2005. Libertar o crédito: como aprofundar e estabilizar o financiamento bancário, Río de Janeiro, Campus.

Cargill, T. (1983), Money, the Financial System and Monetary Policy, Englewood Cliffs, New Jersey, Prentice-Hall.

Carvalho, F.J. Cardim de (2010), "Reformas financeiras para apoiar o desenvotlvimento", Textos para Discussão CEPAL-IPEA, $\mathrm{N}^{\circ} 14$ (LC/BRS/R.231), Brasilia, Comisión Económica para América Latina y el Caribe (CEPAL)/Instituto de Investigación Económica Aplicada (IPEA).

(2009), "Systemic crisis, systemic risk and the financial instability hypothesis", Macroeconomic Policies on Shaky Foundations, E. Hein, T. Niechoj y E. Stockhammer (eds.), Berlín, Metropolis-Verlag.

(2005), "Inovação financeira e regulação prudencial: da regulação de liquidez aos Acordos de Basiléia", Regulação financeira e bancária, R. Sobreira (org.), São Paulo, Editora Atlas.

(1997), "Financial innovation and the post Keynesian approach to the "process of capital formation"', Journal of Post Keynesian Economics, vol. 19, № 3, M.E. Sharpe.

Dymski, G.A. (2006), "Discrimination in the credit and housing markets: findings and challenges", Handbook on the Economics of Discrimination, William Rodgers (ed.), Cheltenham, Reino Unido, Edward Elgar.

(2005), "A eficiência social e a regulação bancária: lições da experiência americana”, Regulação financeira e bancária, R. Sobreira (org.), São Paulo, Editora Atlas.

Fry, M.J. (1995), Money, Interest and Banking in Economic Development, Londres, Johns Hopkins University Press.

Furtado, C. (1976), Teoría y política del desarrollo económico, México, D.F., Siglo XXI.

Gerschenkron, A. (1962), Economic Backwardness in Historical Perspective: A Book of Essays, Cambridge, Massachusetts, Belknap Press of Harvard University Press.

Gurley, J. y E. Shaw (1960), Money in a Theory of Finance, Washington, D.C., Brookings Institution.

(1955), "Financial aspects of economic development", The American Economic Review, vol. 45, ํㅜ 4, Nashville, Tennessee, American Economic Association, septiembre.

Hall, B.H. (2002), "The financing of research and development", Working Papers, $\mathrm{N}^{\circ}$ E01-311, Berkeley, Universidad de California en Berkeley, enero.

Hermann, J. (2011a), "Política financeira e desenvolvimento econômico: um debate a partir de contribuições recentes de Fernando Cardim de Carvalho", Anais do IV Encontro Internacional da Associação Keynesiana Brasileira, Río de Janeiro, Instituto de Economía, Universidad Federal de Río de Janeiro, agosto.

(2011b), "O mercado de títulos privados e a inadequação da curva de rendimentos no Brasil: análise teórica e da experiência pós-estabilização (1995-2010)", Working Paper, $\mathrm{N}^{\circ}$ 14, Banco de Desarrollo del Brasil (BNDES)/Asociación Nacional de Centros de Posgrado en Economía (ANPEC).

(2004), "O trade off do crescimento no Brasil nos anos 1990-2000: análise crítica e alternativas de política monetária", Revista Econômica, vol. 6, N2 2, Río de Janeiro, diciembre.

(2003), "O modelo de liberalização financeira dos anos 1990: 'restatement' ou auto-crítica?", Nova Economia, vol. 13, $\mathrm{N}^{\circ}$ 2, Belo Horizonte, Universidad Federal de Minas Gerais, julio-diciembre.

Herring, R.J. y N. Chatusripitak (2000), The Case of the Missing Market: The Bond Market and Why It Matters for Financial Development, Tokio, Wharton Financial Institutions Center, mayo.
Keynes, J.M. (1943), Teoría general de la ocupación, el interés y el dinero, México, D.F., Fondo de Cultura Económica.

(1937a), "Alternative theories of the rate of interest", The Economic Journal, Hoboken, New Jersey, Wiley, junio.

(1937b), "The 'ex-ante theory' of the rate of interest", The Economic Journal, Hoboken, New Jersey, Wiley, diciembre.

Kregel, J. (1997), "Margins of safety and weight of the argument in generating financial fragility", Journal of Economic Issues, vol. 31, $\mathrm{N}^{\circ} 2$, Association for Evolutionary Economics, junio. (1986), "A note on finance, liquidity, saving, and investment", Journal of Post Keynesian Economics, vol. 9, $\mathrm{N}^{\circ}$ 1, Armonk, M.E. Sharpe.

(1980), "Markets and institutions as features of a capitalistic production system", Journal of Post Keynesian Economics, vol. 3, $\mathrm{N}^{\circ} 1$, Armonk, M.E. Sharpe.

Malkiel, B.G. (1994), "Efficient market hypothesis", The New Palgrave Dictionary of Money and Finance, P. Newman, M. Milgate y J. Eatwell (eds.), Londres, MacMillan Press Limited.

Matouk, J. (2010), "Financing innovation: an historical approach", Journal of Innovation Economics, vol. 6, $\mathrm{N}^{\circ}$ 2, Universidad De Boeck.

McKinnon, R. (1973), Money and Capital in Economic Development, Washington, D.C., The Brookings Institution.

Minsky, P.H. (1986), Stabilizing an Unstable Economy, New Haven, Yale University Press.

(1982), "The financial fragility hypothesis: an interpretation of Keynes and an alternative to 'standard' theory", Can It Happen Again? Essays on Stability and Finance, H.P. Minsky, Nueva York, M.E. Sharpe, Inc.

Obstfeld, M. (1994), "The logic of currency crises", NBER Working Paper, $\mathrm{N}^{\circ} 4640$, Cambridge, Massachusetts, National Bureau of Economic Research.

Schumpeter, J.A. (1934), The Theory of Economic Development, Cambridge, Massachusetts, Harvard University Press.

Shaw, E.S. (1973), Financial Deepening in Economic Development, Nueva York, Oxford University Press.

Sinapi, C. (2011), "Institutional prerequisites of financial within Minsky's financial instability hypothesis: a proposal in terms of 'institutional fragility', Working Paper, $\mathrm{N}^{\circ}$ 674, Dijon, Levy Economics Institute of Bard College, julio.

Stiglitz, J. (1994), "The role of the state in financial markets", Proceedings of the World Bank Annual Conference on Development Economics 1993, Washington, D.C., Banco Mundial.

Stiglitz, J. y A. Weiss (1981), "Credit rationing in markets with imperfect information", American Economic Review, vol. 71, $\mathrm{N}^{\circ}$ 3, Nashville, Tennessee, American Economic Association, junio.

Studart, R. (2005), "Financiamento do desenvolvimento", Economia Brasileira Contemporânea: 1945-2004, F. Giambiagi y otros (orgs.), Río de Janeiro, Campus.

(1995), Investment Finance in Economic Development, Londres, Routledge.

(1995-96), "The efficiency of financial system, liberalisation and economic development", Journal of Post Keynesian Economics, vol. 18, $\mathrm{N}^{\circ} 2$, M.E. Sharpe Inc.

Tavares, M. da C. (1979), Da substituição de importações ao capitalismo financeiro, Río de Janeiro, Zahar Editores.

Tobin, J. (1958), "Liquidity preference as behavior toward risk", The Review of Economic Studies, vol. 25, ํㅜㄹ. Oxford University Press, febrero.

Zysman, J. (1983), Governments, Markets and Growth, Londres, Cornell University Press. 